\title{
Main problems associated with obtaining informed consent of cardiologic patients for participation in scientific studies: Focus on acute care
}

\author{
Beata Morawiec ${ }^{1}$, Damian Kawecki ${ }^{1 *}$ and Lesław Niebrój ${ }^{2}$ \\ ${ }^{1}$ 2nd Department of Cardiology, School of Medicine with the Division of Dentistry, Zabrze, Medical University of Silesia, Katowice, Poland \\ ${ }^{2}$ Department of Philosophy and Human Sciences, Medical University of Silesia, Katowice, Poland
}

\begin{abstract}
In the era of evidence based medicine, progress in cardiology requires continuous inflow of scientific data that support new standards and newly-formulated hypotheses. However, cardiologic research should adhere to the same ethical principles as studies in any other discipline. The aim of this paper is to review current issues that raise most concerns and ethical problems in cardiologic research. Important cardiology-specific ethical issue is the research on acute coronary syndromes. Life-threatening conditions markedly impair patient's assessment of the situation and put constant time pressure on treating physician. Therefore, the patient is capable of only simple choices between expressing non-informed consent and lack thereof. One can hardly determine if recruitment of study participants represents a sufficient reason for disruption in one's autonomy. Achieving this objective will not be possible without progress in science and involvement of patients, who should be active participants through expressing their informed consent for participation in research.
\end{abstract}

\section{Introduction}

Cardiology is one of the most dynamic and variable medical disciplines. As such it stimulates continuous development of novel technologies, diagnostic and therapeutic modalities, which is reflected by constantly updated guidelines on patient management. In the era of evidence based medicine, progress in cardiology requires continuous inflow of scientific data that support new standards and newly-formulated hypotheses. As any other healthcare specialists, cardiologists are expected to adhere to highly standardized diagnostic and therapeutic guidelines that are developed by international consensus groups on the basis of evidence from large clinical trials. A search of the international clinical trial registry run by the U.S. National Institutes of Health returned 2,024 hits for the term "cardiology" [1]. The application of such guidelines for treatment of individual patients always requires good clinical judgment and discretion, and, first and foremost, commitment to ethical standards [2]. Apart from the evidence-based guidelines, management of cardiologic patients should be also based on the principles of professional morality expressed in internationally recognized documents (e.g. Good Clinical Practice (GCP) and country-specific Codes of Medical Ethics. However, application of the ethical principles to a highly variable population of cardiologic patients, including both pediatric and geriatric subjects, who may require either immediate intervention (e.g. in the case of sudden cardiac arrest or myocardial infarction) or treatment of a chronic condition (e.g. end-stage heart failure), becomes highly challenging. Moreover, discussing ethical issues in cardiology, one should consider specific character of clinical studies in this discipline. Nevertheless, cardiologic research should adhere to the same ethical principles as studies in any other discipline of clinical medicine. The aim of this paper is to review current issues that raise most concerns and ethical problems in cardiologic research.

\section{General problems}

One can hardly identify ethical issues that are highly specific for cardiology, as most of them raise a number of concerns in other medical disciplines as well. On the other hand, however, moral dilemma in cardiology are distinguished by the very nature of cardiac diseases, the treatment used and socially accepted assumptions related both to these diseases as well as to conceptualization of hearth as an image or symbol of human emotions etc. [3]. Accordingly, in our opinion, the list of cardiology-specific ethical problems includes care of pediatric patients and genetically predisposed individuals, readability and clarity of information presented in the informed consent forms, and scientific jargon that may be incomprehensible to a lay person [4-6]. The importance of the two latter issues was well documented in a study analyzing understanding of the informed consent process by participants of one randomized placebo controlled trial. As many as $98 \%$ of patients (among them individuals with cardiologic problems) were satisfied with the process of obtaining informed consent. However, most of them, irrespective of educational level, did not understand the role of randomization and the reason for placebo use [7]. This suggests that patients may create their own systems of comprehensibility assessment. Consequently, implementation of objective assessment criteria of the informed consent process seems of vital importance, as a

Correspondence to: Damian Kawecki, 2nd Department of Cardiology in Zabrze, Medical University of Silesia, M. Curie-Skłodowskiej 10, 41-800 Zabrze, Poland, Tel: +48 322711 010; E-mail: d.kawecki@interia.pl

Keywords: informed consent, clinical trials, provisional consent, sudden conditions, cardiology

Received: September 08, 2016; Accepted: October 05, 2016; Published: October 07,2016 
free, non-enforced decision and complete understanding of presented information represent prerequisites of truly informed consent $[8,9]$. Analyzing potential difficulties in obtaining informed consent, one should not focus solely on patient-derived reasons, such as clinical or personal issues. The role of the physicians should be considered, as a person who could not give their patients opportunity to consent, provide them with inadequate information, or make involuntary or even purposeful attempts to influence their decision by coercion, persuasion and/or manipulation) [9-12].

\section{Specific problems of cardiologic patients}

The research on acute coronary syndromes, including ST elevation myocardial infarction (STEMI), represents a cardiology-specific ethical issue. While the objectives of these studies do not raise per se major ethical concerns, the right for obtaining informed consent for participation in such trial from a patient who requires immediate intervention is less obvious [13]. Each consent can be put into question if a patient's ability to ask questions was limited or if a physician was unable to address all the patient's concerns. Such limitations undoubtedly apply to sudden medical conditions (the effects of the acute illness, time constraints, lack of/limited access to family and other significant person etc.) [14]. Usually, a patient with myocardial infarction remains in a "gray zone" between resuscitation attempts and constructive discussion of a study protocol under stable conditions. For further considerations, let's assume a situation of obtaining an informed consent for participation in a study which was approved by the Local Bioethics Committee and requires immediate enrollment of participants. Does a consent that was obtained under life-threatening conditions and time pressure really represent the informed consent? What a delay in the implementation of a guideline-recommended treatment can be accepted as a result of analysis of one's eligibility to a study? These two questions have been asked for a long period of time. Modern technologies, as well as diagnostic and therapeutic guidelines on management of STEMI patients were developed as a result of a number of clinical trials that have been conducted for more than 20 years. The authors of these trials were well aware of the fact that time is crucial for the management of myocardial infarction. However, previous legal regulations of clinical trials did not address the issue of obtaining informed consent from an unconscious patient with a sudden medical condition. Therefore, clinicians used several avoidance techniques, such as refraining from this type of trials, skipping the informed consent process, or obtaining modified or provisional consent. Also an alternative form of a twostage informed consent process, allowing continuation of a clinical trial with simultaneous protection of patient rights, was proposed [15]. The correctness of this latter attitude was verified several years later in a group of patients with myocardial infarction and unstable angina pectoris. The understanding of a study protocol and enrollment criteria by the participants turned out to be better on the second assessment (24 h vs. $10 \mathrm{~h}$ after randomization) [16]. According to the current International Ethical Guidelines for Biomedical Research Involving Human Subjects, developed in Geneva by the Council for International Organizations of Medical Sciences (CIOMS) in collaboration with the World Health Organization (WHO), a provisional consent from a patient's family member can be obtained, providing that the final consent form is completed by the patient promptly thereafter [8]. Similar concept was presented in the document published by the U.S. Food and Drug Administration (FDA) [17]. However, the exact definition of a "family member" still remains an open question [18]. Furthermore, the procedure of referral to third parties is hardly acceptable in cases in which time is a crucial issue. According to a common saying, "time is muscle" (i.e. myocardium). Previously published study showed that the process of obtaining informed consent from STEMI patients causes an alarmingly, average 11-minute delay in implementation of all necessary therapeutic procedures [19]. According to the abovementioned Geneva Guidelines, a delay in the implementation of treatment is acceptable whenever additional procedures (e.g. additional blood sampling) are required by a study protocol. However, the latter procedures should constitute a merit of a study and a reason for obtaining participant's consent. Nevertheless, the delays in treatment implementation result in an evident disobedience of respective therapeutic guidelines. The use of exception from informed consent (EFIC) is limited to studies dealing with resuscitation and therapeutic interventions in unconscious individuals [20]. Although EFIC proved to be widely accepted by the patients [21,22], extrapolation of this form on conscious subjects with myocardial infarction seems unlikely due to considerable inconsistency of interpretation criteria [23]. Due to objective barriers in form of emotional and somatic stress associated with the acute cardiologic condition, one is less likely to express a truly informed consent. As the patients with myocardial infarction show far greater knowledge of benefits than of risks associated with a given trial [16], the use of informed refusal procedure was postulated [24], thus making the consent a virtually implied issue.

Life-threatening conditions, such as STEMI, markedly impair a patient's assessment of the situation. Therefore, the patient is capable of only simple choices between expressing non-informed consent and lack thereof. One can hardly determine if recruitment of study participants represents a sufficient reason for disruption in one's autonomy. Undoubtedly, among the ethical rules, which are supported by the principle of the respect for autonomy, the rule "Obtain the consent for any intervention with patient" plays a crucial role in the contemporary medicine [14]. On the other hand, it should be emphasized that so called expressed or explicit consent is, though paradigmatic, the only one among different ways to obtain patient's consent. Cardiologists are under constant pressure to reduce incidence and mortality rates of cardiovascular conditions as the main cause of fatal outcomes worldwide. Achieving this objective will not be possible without progress in science and involvement of patients, who should be active participants of the process through expressing their informed consent for participation in research.

\section{References}

1. US National Institutes of Health (2016) ClinicalTrials.gov.

2. Barthelemy N, Herman M, Boga D, Princen F, Thirion C, et al. (2014) What is the place of humans within the recommendations of the multidisciplinary oncology consultations and therapeutic project? Review Medicale de Liege 69 Suppl 1: 9-12.

3. Alberti FB (2010) Matters of the Heart: History, Medicine, and Emotion. Oxford-New York: Oxford University Press.

4. Merlo DF, Knudsen LE, Matusiewicz K, Niebrój L, Vähäkangas KH (2007) Ethics in studies on children and environmental health. J Med Ethics 33: 408-413. [Crossref]

5. Terranova G, Ferro M, Carpeggiani C (2012) Low quality and lack of clarity of current informed consent forms in cardiology: how to improve them. JACC Cardiovasc Imaging 5: 649-655. [Crossref]

6. Meneguin S, Zoboli EL, Domingues RZ, Nobre MR, César LA (2010) Informed consent as viewed by patients participating in cardiology drug trial. Arq Bras Cardiol 94: 4-9. [Crossref]

7. Pope JE, Tingey DP, Arnold JM, Hong P, Ouimet JM, et al. (2003) Are subjects satisfied with the informed consent process. A survey of research participants. J Rheumatol 30: 815-824. [Crossref]

8. Idänpään-Heikkilä JE1, Fluss S (2004) The CIOMS view on the use of placebo in clinical trials. Sci Eng Ethics 10: 23-28. [Crossref] 
9. Beauchamp TL, Childress JF (2013) Principles of Biomedical Ethics, Oxford-New York: Oxford University Press.

10. Borracci RA, Calderón G, Seoane MR, Perez AC, Doval HC (2008) Ethical review and informed consent in cardiovascular research reports in Argentina. Arq Bras Cardiol 90: 290-293. [Crossref]

11. DeLuca SA, Korcuska LA, Oberstar BH, Rosenthal ML, Welsh PA, et al. (1995) Are we promoting true informed consent in cardiovascular clinical trials. J Cardiovasc Nurs 9: 54-61. [Crossref]

12. Hoerni B, Saury R (1998) The consent. Information, autonomy and decision in medicine. Paris: Masson.

13. The Task Force on the management of ST-segment elevation acute myocardial infarction of the European Society of Cardiology (ESC) (2012) ESC Guidelines for the management of acute myocardial infarction in patients presenting with ST-segment elevation. European Heart Journal 33: 2569-619.

14. Limkakeng AT, de Oliveira LL, Moreira T, Phadtare A, Garcia Rodrigues C et al (2014) Systematic review and metasummary of attitudes toward research in emergency medical conditions. Journal of Medical Ethics 40: 401-408.

15. Grim PS, Singer PA, Gramelspacher GP, Feldman T, Childers RW, et al. (1989) Informed consent in emergency research. Prehospital thrombolytic therapy for acute myocardial infarction. JAMA 262: 252-255. [Crossref]

16. Kucia AM, Horowitz JD (2000) Is informed consent to clinical trials an "upside selective" process in acute coronary syndromes. Am Heart $J$ 140: 94-97. [Crossref]
17. US Food and Drug Administration (2013) Code of Federal Regulations, 21CFR50.24, Exceptions from informed consent requirements for emergency research.

18. Moutel G (2003) Consent in pratiqeus care and medicine research. Between idealism and clinical realities. Paris, L'Harmattan.

19. Blankenship JC, Skelding KA, Scott TD, Buckley J, Zimmerman DK, et al. (2009) ST-elevation myocardial infarction patients can be enrolled in randomized trials before emergent coronary intervention without sacrificing door-to-balloon time. Am Heart $J$ 158: 400-407. [Crossref]

20. Levine RJ (1986) Ethics and regulation of clinical research. (2nd Edn) Baltimore, Munich: Urban \& Schwarzenberg.

21. Dickert NW, Mah VA, Baren JM, Biros MH, Govindarajan P, et al. (2013) Enrollment in research under exception from informed consent: the Patients' Experiences in Emergency Research (PEER) study. Resuscitation 84: 1416-1421. [Crossref]

22. Dickert NW, Kass NE (2009) Patients' perceptions of research in emergency settings a study of survivors of sudden cardiac death. Social Science \& Medicine 68: 183-191.

23. Ernst AA, Fish S (2005) Exception from informed consent: viewpoint of institutional review boards - balancing risks to subjects, community consultation, and future directions. Academic Emergency Medicine 12: 1050-1055.

24. Dickert NW, Llanos A, Samady H (2012) Re-visiting consent for clinical research on acute myocardial infarction and other emergent conditions. Progress in Cardiovascular Diseases 55: 251-257.

Copyright: $@ 2016$ Morawiec B. This is an open-access article distributed under the terms of the Creative Commons Attribution License, which permits unrestricted use, distribution, and reproduction in any medium, provided the original author and source are credited. 